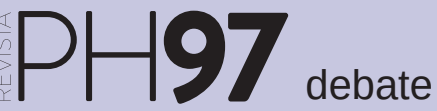

a debate El papel del patrimonio en las nuevas políticas para la cultura, la ciudad y el territorio:

las agendas urbanas como marco de oportunidad

| coordinan Blanca del Espino Hidalgo, Rafael Merinero Rodríguez

\title{
Breve síntesis ¿Están el patrimonio y la cultura en la agenda de las ciudades del futuro?
}

\author{
Rafael Merinero Rodríguez | Departamento de Sociología, Universidad Pablo de Olavide \\ Blanca del Espino Hidalgo | Centro de Inmuebles, Obras e Infraestructuras, IAPH
}

URL de la contribución <www.iaph.es/revistaph/index.php/revistaph/article/view/4438>

El Debate número 13 de la revista $\mathrm{PH}$ plantea una discusión abierta y crítica sobre en qué manera y medida el patrimonio cultural interviene, ya sea como objeto, como herramienta o como recurso, en las políticas urbanas, culturales y territoriales más recientes, presentes y futuras. Las distintas contribuciones recibidas muestran un acercamiento plural en el que se entrelazan visiones y disciplinas muy diversas, con ciertos argumentos que funcionan como un hilo conductor en el que el que el posicionamiento del autor respecto a los procesos de transformación de la ciudad, pero también hacia el rol del patrimonio cultural en el contexto urbano-territorial, condiciona la reflexión respecto a otras cuestiones fundamentales que aparecen de manera recurrente como la participación ciudadana, la apropiación del espacio, la identidad o el desarrollo sostenible.

El subtítulo propuesto para el debate sugería la oportunidad de su desarrollo a partir de la emergencia de una nueva figura en el ámbito de la planificación: las agendas urbanas. Esta herramienta ha cobrado un especial vigor recientemente, en especial a partir de la Conferencia de las Naciones Unidas (ONU) sobre la Vivienda y el Desarrollo Urbano Sostenible Habitat III, celebrada en Quito (Ecuador) en octubre de 2016 (NACIONES UNIDAS, 2017), que definió la Nueva Agenda Urbana (NUA) con un horizonte de aplicación de dos décadas.

Además, el documento acordado en el marco internacional se produce en relación con la Agenda 2030 para el Desarrollo Sostenible (NACIONES UNIDAS, 2015) que había sido aprobada un año antes y, en particular, para la aplicación del Objetivo 11: Ciudades y comunidades sostenibles, haciendo explícita esta conexión en varias ocasiones dentro del texto.
En este sentido, ya se ponían de manifiesto en el texto introductorio al debate los vínculos entre las temáticas abordadas en los grandes eventos recientes relacionados con las políticas urbanas de sostenibilidad y los relativos a los retos del patrimonio cultural. Así pues, el valor social del patrimonio ha sido constatado en diferentes declaraciones mundiales ${ }^{1} y$, de manera creciente, esta cuestión ha ido tomando peso respecto a las miradas exclusivamente monumentalistas, historicistas o mercantilistas sobre el mismo.

Por otra parte, las declaraciones más recientes incorporan ya la noción de sostenibilidad, destacando entre ellas la Declaración de Hangzhou de la ONU en 2013 Situar la cultura en el centro de las políticas de desarrollo sostenible ${ }^{2}$ (NACIONES UNIDAS, 2013), que ponía sobre la mesa el papel fundamental que el patrimonio cultural ha tenido, en los años posteriores, en la formulación de directrices y la elaboración de documentos de planificación a escala mundial. Tras ella, se sucederán los documentos que desembocarán, tanto desde el ámbito del patrimonio (UNESCO y organismos asociados) ${ }^{3}$ como desde las instituciones que han liderado la evolución en los principios de sostenibilidad (fundamentalmente en hitos temporales promovidos por Naciones Unidas) en su aplicación tanto en lo global como en lo local, en la formulación de la mencionada Nueva Agenda Urbana y de los 17 Objetivos de Desarrollo Sostenible (ODS) presentes en la Agenda 2030. Nos encontramos, pues, ante un campo de trabajo en constante evolución y que, desde sus inicios, ha tenido entradas tanto desde el ámbito de la cultura y del patrimonio como desde las directrices de la sostenibilidad aplicadas al territorio. Como se constata de la lectura de las diferentes aportaciones al presente Debate, este enfoque dual con constantes puntos de 
a debate El papel del patrimonio en las nuevas políticas para la cultura, la ciudad y el territorio:

las agendas urbanas como marco de oportunidad

| coordinan Blanca del Espino Hidalgo, Rafael Merinero Rodríguez

interacción se mantiene como una dinámica claramente apreciable en la actua ${ }^{4}$ los elementos centrales del discurso, comenzaremos por hacer alusión a la presencia del patrimonio y la cultura en las Agendas Urbanas que se presentan aquí como espacio o momento de oportunidad para la llamada a contribuciones. En efecto, la propia discusión sobre estos instrumentos ha centrado gran parte de ellas en aspectos a los que haremos alusión más adelante, entre otros, su carácter no vinculante como elemento de planificación, la metodología de participación asociada a ellas o su aplicación y particularización en la escala local. Así, el encaje del patrimonio cultural tanto en las agendas urbanas como en los ODS se revela, tal como se advertía en el texto introductorio y han constatado varios de los autores, como una cuestión transversal o de fondo más que como un objetivo fundamental en sí mismo.

La manera en la que los diferentes documentos de Agenda Urbana incorporan esta cuestión difiere de unos a otros, si bien mantiene ciertos puntos en común: aparece, de forma innegable, la cuestión del uso productivo de los bienes patrimoniales como un recurso, ya sea para el desarrollo local o, en la mayoría de los casos, relacionados con el turismo cultural como uno de los grandes sectores productivos de nuestro tiempo. Sin embargo, en el caso de los ODS, será precisa esperar a las Metas desarrolladas a posteriori para encontrar líneas de trabajo específicas a la protección, la acción o el uso de la cultura y el patrimonio para la obtención de los grandes retos.

La Nueva Agenda promulgada a partir de Habitat III atará, de manera inequívoca y posiblemente condicionando los desarrollos futuros a escalas menores, la aplicación y la formulación de retos de sostenibilidad a la cuestión espacial y territorial. La cultura y el patrimonio van a ser entendidas dentro de la Declaración de Quito en una triple concepción: como recurso para el desarrollo -con objeto fundamentalmente crematístico, es decir, mediante las posibilidades de una mejora de las condiciones socioeconómicas de las ciudades a partir de su explotación-, como objeto de protección

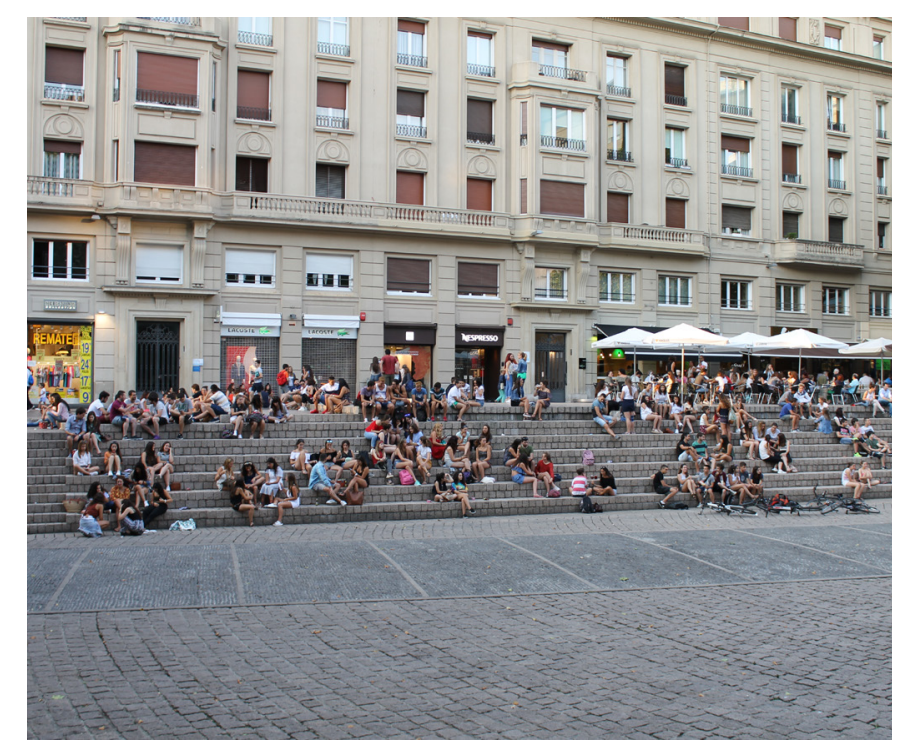

Apropiación social de la ciudad y su espacio público. Plaza de los Fueros, Vitoria (agosto, 2016) | Blanca del Espino Hidalgo

-es decir, entendidos los bienes del patrimonio cultural y natural como un acervo en riesgo- y, por último, en base a su posible función como estímulo de la participación, la responsabilidad o la innovación. Esta última acepción queda esbozada en el punto 125 de la Declaración, considerándose como parte de la sección dedicada a Planificación y gestión del desarrollo espacial urbano, es decir, más vinculada al uso o tratamiento de los espacios físicos construidos y no construidos de la ciudad que a cuestiones referidas a la creatividad y la innovación social.

Descendiendo en ámbito de aplicación, la Agenda Urbana para la Unión Europea (Unión Europea, 2016) se elabora a partir de la publicación del Pacto de Ámsterdam, un documento genérico enfocado hacia grandes retos como la regeneración, el desarrollo o la cohesión que no hace alusión específica al patrimonio, si bien menciona la cultura de manera tangencial. Sin embargo, el primer hito temporal en la implementación de la agenda europea, que supone la creación de partenariados para la consecución de objetivos precisos en diferentes ámbitos temáticos, cuenta ya con uno relativo a Cultura y Patrimonio Cultural, aprobado en noviembre 
a debate El papel del patrimonio en las nuevas políticas para la cultura, la ciudad y el territorio:

las agendas urbanas como marco de oportunidad

| coordinan Blanca del Espino Hidalgo, Rafael Merinero Rodríguez

de 2018, que integra tanto gobiernos nacionales como instituciones regionales o locales y está liderado por Alemania e Italia.

En cuanto a las agendas urbanas con un carácter territorial de menor escala, la Agenda Urbana Española enfatiza el tratamiento del patrimonio cultural y natural como bienes a proteger y conservar, sin hacer un excesivo hincapié en sus posibilidades como recurso para el desarrollo o la cohesión social, con la excepción de las posibilidades que presentaría el turismo cultural patrimonial. Por su parte, en la Agenda Urbana de Andalucía (JUNTA DE ANDALUCÍA, 2018), elaborada con anterioridad a la nacional y la que hasta hoy es la única agenda urbana aprobada de ámbito regional en España, se propone el patrimonio urbano y territorial como objeto de protección y regeneración, pero también como fuente del hábitat sostenible. Al mismo tiempo, se constata una preocupación por la mercantilización de la ciudad histórica y su excesiva turistificación en detrimento de la habitabilidad de este tejido urbano y la disponibilidad de vivienda para la ciudadanía. En el caso andaluz, además, los retos relacionados con el patrimonio se asocian fundamentalmente a la dimensión espacial al igual que ocurría con la NUA mientras que, en otros, como los ODS, aparecen distribuidos a lo largo de varios de los grandes diecisiete objetivos.

La cuestión de la escala y la aplicabilidad de las agendas urbanas en este sentido es, de hecho, una de las ideas que más notoriedad han adquirido en las distintas contribuciones a este Debate. Así pues, se denota un especial énfasis hacia la escala local y el papel de las administraciones municipales en el ejercicio y la formulación de políticas concretas para el futuro de las ciudades y la potenciación del rol del patrimonio cultural en su consecución. Esto constata una tendencia creciente hacia considerar lo urbano -o la ciudad-como el gran objeto de debate y trabajo en el ámbito actual y próximo de los nuevos mecanismos de planificación. Las miradas que se apuntan en este sentido son diversas desde un punto de vista inmediato: desde el uso de los recursos patrimoniales como motor de desarrollo local hasta la posibilidad de crear herramientas efectivas de localización, protección, puesta en marcha y valoración de los bienes.

Sin embargo, un discurso de base se entrelaza lo largo de las aportaciones realizadas y pone de manifiesto una de las grandes inquietudes actuales en la formulación de herramientas de carácter urbano-territorial: la necesidad de adaptación y concreción de los principios, métodos y retos a cada escala y también a cada caso en función de sus características particulares. Se pone en crisis, así, el carácter excesivamente general y no vinculante de la Nueva Agenda Urbana global, la establecida para la Unión Europea e, incluso, la de Andalucía, revelando como prioridad actual la elaboración otras de carácter local que atiendan a los hechos y retos de cada ciudad, sus características y su escala, lo que por lo general aún no se producido, salvo en casos singulares como el de Málaga.

De hecho, uno de los principales motivos de controversia acerca del concepto de agenda urbana en sí mismo ha sido el propiciado por el debate de lo rural, por entenderse fuera de contexto los núcleos de menor tamaño en la consideración de lo urbano entendido como apelativo estricto, pero también fundamentado en la inquietud sobre la despoblación de ciertas áreas de España y Europa, así como de algunos territorios andaluces.

El potencial que los recursos patrimoniales, tanto por su aprovechamiento para el desarrollo socioeconómico como por su contribución al equilibrio ambiental o al fomento de una identidad que contribuya al anclaje de la población, ha sido revelado en varios de los textos presentados, en los que también se advierte la necesidad de trabajar en una escala más allá de lo local o de los límites administrativos estrictamente establecido para crear redes de municipios que, basadas en un motivo o un concepto patrimonial común, establezcan estrategias conjuntas asociadas al territorio, añadiendo al ya consabido paradigma de lo global desde lo local uno nuevo, que se acercaría más a una autonomía local interconectada y, a su vez, conectada al territorio. 
a debate El papel del patrimonio en las nuevas políticas para la cultura, la ciudad y el territorio: las agendas urbanas como marco de oportunidad | coordinan Blanca del Espino Hidalgo, Rafael Merinero Rodríguez

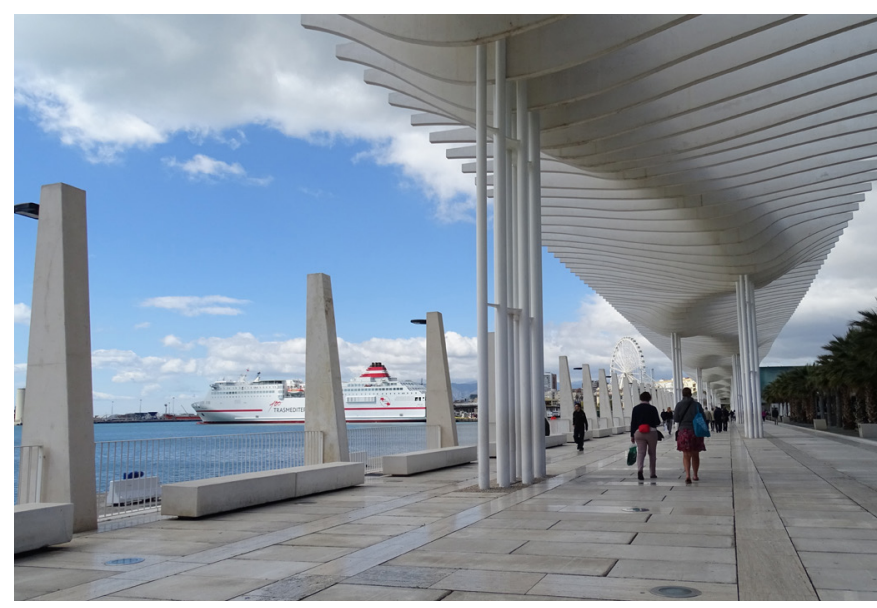

Regeneración urbana en el puerto de Málaga. Muelle 1, 2017 | fuente pxhere

Profundizando en las estrategias de construcción de la ciudad y de elaboración de las propias agendas, la gobernanza ocupa, indudablemente, otro de los lugares centrales del discurso. Por una parte, debe ser entendido que la situación del ODS 17, dedicado al establecimiento de alianzas para conseguir el resto de objetivos, como cierre de la Agenda 2030, manifiesta una declaración de intenciones que refrenda lo ya comentado anteriormente: la colaboración entre instituciones y administraciones es fundamental para el desarrollo e implementación de las políticas urbanas, culturales o patrimoniales. Del otro lado, sin embargo, aparece la participación ciudadana como uno de los argumentos que con mayor frecuencia surgen en la formulación de cualquier tipo de documento de planificación y que, a la ver, suscita más polémica, lo que tiene un reflejo manifiesto en muchas de las contribuciones realizadas en este debate.

En este contexto, se propone a la sociedad como la auténtica protagonista de las nuevas agendas urbanas, más allá del énfasis ya prestado tradicionalmente a la planificación espacial. Además de esto, se pone en crisis el modelo según el cual la comunidad urbana es escuchada y se implica de cara a la elaboración de diagnósticos y propuestas así comel papel de las instituciones $y$ asociaciones que funcionan, a menudo, como intermediarios entre la elevación de las problemáticas y propuestas desde el ciudadano particular-lo que se conoce

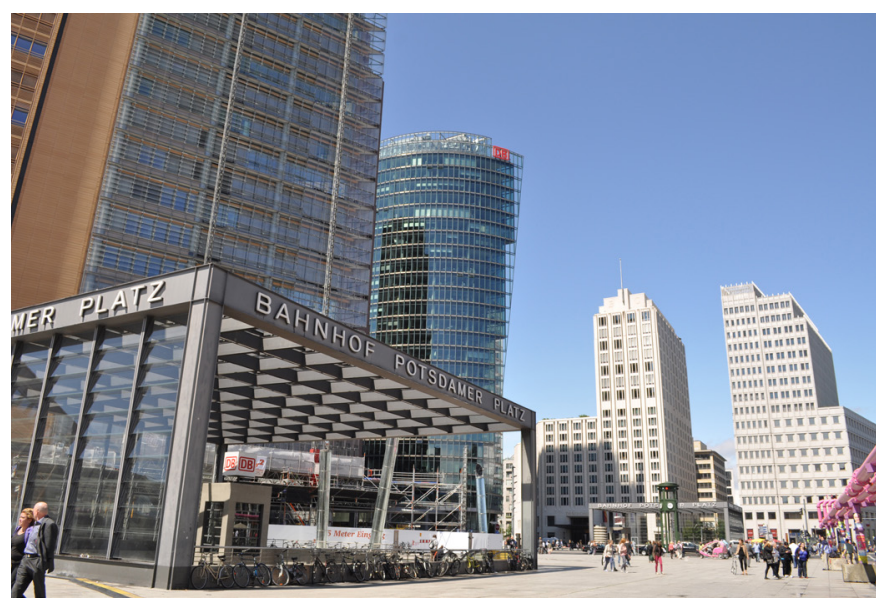

Regeneración urbana en Potsdamer Platz, Berlín. 2012 | foto Blanca del Espino Hidalgo

como estrategia bottom-up- y la promoción de los grandes documentos desde los organismos de toma de decisiones -a la que se aplica el anglicismo top-down-, a los que se recrimina una desconexión generalizada con la realidad cotidiana y se propone mejorar y ampliar los métodos de consulta popular con la inclusión de las tecnologías digitales o los relatos urbanos como herramientas de comunicación y acceso.

Sin embargo, y especialmente dentro del discurso que nos ocupa en esta ocasión, la cultura y el patrimonio aparecen también como protagonistas en el momento de tratar la participación ciudadana, principalmente vinculados al concepto de identidad patrimonial, es decir, el aprovechamiento del sentimiento de comunidad avalado por memorias, manifestaciones culturales y símbolos identitarios compartidos que contribuye, en primer lugar, a la sensibilización sobre las cuestiones que atañen al entorno urbano $y$, tras ello, a la formulación de líneas de trabajo específicas que deben ser incorporadas a las agendas urbanas y otros instrumentos de planificación.

A este respecto, la educación se convierte en una baza fundamental para el fomento tanto de la identidad patrimonial como de la cultura participativa entre la población. Esto no se limita únicamente a la educación de las generaciones más jóvenes -que serán, ciertamente, 
a debate El papel del patrimonio en las nuevas políticas para la cultura, la ciudad y el territorio:

las agendas urbanas como marco de oportunidad

| coordinan Blanca del Espino Hidalgo, Rafael Merinero Rodríguez

las protagonistas y responsables de la sostenibilidad y el patrimonio del mañana- sino, como ya establecía la carta de Aalborg 5 (Conferencia Europea, 1994), también a la formación de la ciudadanía en general y de las personas encargadas de tomar las decisiones, formular e implementar las políticas sobre la planificación urbana y territorial.

Además de al fomento de la participación a partir de la conciencia patrimonial, diferentes contribuciones apuntan al entendimiento del patrimonio como recurso para cuestiones no directamente ligadas al desarrollo urbano, sino más vinculadas a principios que sería necesario fortalecer e incorporar a las propias agendas urbanas o a sus mecanismos de implementación. Son ejemplos de esto la cuestión del género y la inclusividad contra la segregación social en las ciudades o el patrimonio como mecanismo de apropiación de la ciudad y, más concretamente, del espacio público.

Precisamente, los discursos más recientes sobre la significación social del espacio urbano ponen de manifiesto al espacio público y lo común como los lugares fundamentales de identificación patrimonial en la ciudad en el sentido de una participación física y directa mediante la ocupación del espacio urbano y la manifestación cultural y social en el mismo, en contraposición al ya mencionado espacio virtual como herramienta de difusión, transparencia y fomento de la cultura participativa.

Esta dualidad entre la ciudad como lugar y la ciudad como vivencia es puesta de manifiesto por una obra que, por su aparición reiterada en varias de las contribuciones, debe ser referida aquí: la monografía reciente de Richard Sennett (2018) titulada Construir y habitar. Ética para la ciudad, cuyos argumentos sobre cómo se habita, cómo se construye y, en definitiva, qué es la ciudad -o quiénes son la ciudad- podrían funcionar como marco teórico o hilo conductor para la totalidad de esta sección en la que las agendas urbanas y su actualidad han sido el acicate para un debate más profundo sobre el papel actual y el propio concepto del patrimonio cultural construido por y para la sociedad.
Finalmente, los coordinadores quisieran hacer especial énfasis en una cuestión que parece latir al fondo del debate y que, una vez más, parte del contraste entre dos principios: ¿desarrollo para el patrimonio o patrimonio para el desarrollo? Las nuevas agendas y políticas urbanas, enfocadas en su mayoría hacia el desarrollo para la equidad social, el equilibrio ambiental y la prosperidad económica, parecen coincidir en la voluntad de contribuir a la protección, conservación y mejora de los bienes patrimoniales de los territorios en los que tienen aplicación pero, a su vez, entienden al patrimonio cultural como un recurso para la consecución, directa o indirecta, de los grandes retos y objetivos que se marcan en sus principios, entre los que rara vez se encuentra la propia cultura. Este hecho se refleja, a su vez, en la multiplicidad de enfoques ofrecidos por las distintas contribuciones y que, a menudo, dependen de la posición de cada autor, por su experiencia y postura disciplinar, dentro de este denso y profundo entramado de cuestiones, todas ellas de la mayor relevancia, que muestran la complejidad propia de una cuestión que evoluciona permanentemente, tal y como lo hacen la sociedad y el mundo que esta construye y habita. 
a debate El papel del patrimonio en las nuevas políticas para la cultura, la ciudad y el territorio: las agendas urbanas como marco de oportunidad

| coordinan Blanca del Espino Hidalgo, Rafael Merinero Rodríguez

\section{NOTAS}

1. Podemos considerar como punto de partida el Convenio Marco del Consejo de Europa sobre el valor del patrimonio cultural para la sociedad firmado en Faro el 27 de octubre de 2005 (Consejo de Europa, 2005).

2. Como habrán advertido, el lema mantiene cierta simetría con el título de este Debate, al que se añade la cuestión de las Agendas Urbanas como oportunidad actual.

3. Debería destacarse aquí la Declaración de Florencia (UNESCO, 2015) en la que, de manera literal, se solicitaba la inclusión de la cultura entre las estrategias y políticas para el desarrollo sostenible, previamente a la definición de la Agenda 2030 de la ONU.

4. Los conceptos que fundamentan la gran pluralidad de contribuciones a este Debate aparecen, con gran frecuencia, entrelazados, al igual que las relaciones que se establecen entre ellos, por lo que ha resultado complicado para los coordinadores establecer una secuencia estrictamente lineal. Se piden disculpas de antemano por las posibles reiteraciones en este discurso.

\section{La Carta de Aalborg "Carta de las Ciudades Europeas} hacia la Sostenibilidad" fue firmada en 1994 por la Conferencia Europea sobre Ciudades Sostenibles y supuso el punto de partida por el que las ciudades y unidades territoriales participantes se comprometieron a elaborar la Agenda 21 que, tanto en la escala global como en sus posteriores agendas locales, pueden entenderse como las antecesoras directas de las nuevas agendas urbanas.

\section{BIBLIOGRAFÍA}

- Conferencia Europea (1994) Carta de las Ciudades Europeas hacia la Sostenibilidad. Carta de Aalborg de la Conferencia Europea sobre Ciudades Sostenibles, 27 de mayo de 1994 <http://www.ccre.org/docs/Aalborg03_05_span.pdf> [Consulta: 31/05/2019]

- Consejo de Europa (2005) Convenio marco del Consejo de Europa sobre el valor del patrimonio cultural para la sociedad. Declaración de Faro, 27 de octubre de $2005<$ https://rm.coe. int/16806a18d3> [Consulta: 30/05/2019]

- Junta de Andalucía (2018) Agenda Urbana de Andalucía <http://www.cma.junta-andalucia.es/medioambiente/portal_ web/web/temas_ambientales/urbanismo/agenda_urbana_ andalucia_2030.pdf> [Consulta: 28/05/2019]

- Naciones Unidas (2013) Situar la cultura en el centro de las políticas de desarrollo sostenible. Declaración de Hangzhou, 17 de mayo de 2013 <http://www.unesco.org/new/fileadmin/ MULTIMEDIA/HQ/CLT/pdf/final_hangzhou_declaration_ spanish.pdf> [Consulta: 28/05/2019]

- Naciones Unidas (2015) Agenda 2030 para el Desarrollo Sostenible. Objetivos de Desarrollo Sostenible <https://www. un.org/sustainabledevelopment/es/objetivos-de-desarrollosostenible/> [Consulta: 31/05/2019]

- Naciones Unidas (2017) Nueva Agenda Urbana (versión en español) <http://habitat3.org/wp-content/uploads/NUASpanish.pdf> [Consulta: 31/05/2019]

- Sennet, R. (2018) Building and Dwelling. Ethics for the City. New York: Farraf, Strauss and Giroux, 2018 (edición en español Anagrama, febrero 2019)

- UNESCO (2015) Cultura, creatividad y desarrollo sostenible. Investigación, innovación, oportunidades. Declaración de Florencia, 4 de octubre de 2014: <http://unesdoc.unesco.org/ images/0023/002303/230394s.pdf> [Consulta: 30/05/2019]

- UNIÓN EUROPEA (2016) Urban Agenda for the EU. Pact of Amsterdam <https://ec.europa.eu/futurium/en/system/files/ ged/pact-of-amsterdam_en.pdf> [Consulta: 20/06/2019] 